\title{
Consideration of uncertainty in modelling the behaviour of underground excavations
}

\author{
B. Valley Centre for Excellence in Mining Innovation, Canada \\ P.K. Kaiser Centre for Excellence in Mining Innovation, Canada \\ D. Duff Centre for Excellence in Mining Innovation, Canada
}

\begin{abstract}
Uncertainties and variability are the rule when dealing with data from the natural environment such as in geomechanics. Thus, a reliable design approach must be able to consider uncertainties, to evaluate the probability of occurrence of a given scenario and to take measures to reduce the risk to an acceptable level: reducing risk can involve the narrowing of the uncertainty range (e.g. collection of additional data) and/or the spreading of the support capacity to support demand distributions (e.g. upgrading the support standard). In order to assess the effect of uncertainty, one needs probabilistic tools that allow the propagation of the uncertainty from the input parameters (e.g. rock mass strength, modulus) to the design criteria (e.g. magnitude of convergence, depth of yield zone). However, many probabilistic methods (e.g. MonteCarlo simulations) are difficult to introduce in numerical code due to limitations in computing capacities. The alternative investigated in this paper is the point estimate method (PEM), a computing time efficient probabilistic approach. Despite the simplicity of the method, the results are in many situations satisfyingly accurate when comparing them with the output of Monte-Carlo simulation. Also some limitations of the method are highlighted in this paper, particularly when mixed behaviours occur. However, in general, when used with awareness of the assumptions and potential limitations, the PEM approach offers an attractive and very efficient way of considering uncertainty in FEM analyses. It should lead to a broader use of the probabilistic approach in the mining industry and a better assessment of the reliability level of the design of underground openings.
\end{abstract}

\section{Introduction}

In order to develop a reliable design approach, one must use methods to deal with the statistics of the input parameters and the design criteria (e.g. Harr, 1987, 1989). However, tools usually used in geomechanics like stress analyses (e.g. Finite Element Analyses, FEM) are in essence deterministic (a single set of input parameters leads to a single answer). Also these tools are often computing time intensive and not well-suited for the multiple runs needed for systematic sensitivity analyses or statistical simulation (e.g. Monte-Carlo). That's the reason why the Centre for Excellence in Mining Innovation (CEMI) recently contracted RocScience Inc. (Toronto, Canada) to introduce an alternate method, the Rosenblueth point-estimate method (PEM, Rosenblueth, 1975), a simple, computing efficient probabilistic method, into their FEM software Phase $2^{\mathrm{TM}}$ (this new code will be referred as Phase $2 \mathrm{stat}^{\mathrm{TM}}$ ). While this paper makes use of this new code, the PEM approach can be coupled with any stress analysis method and the presented results are valid independently of which is used. This paper evaluates the robustness of the proposed approach by comparing the results obtained from the PEM/FEM combination with outputs from other types of statistical simulations.

When considering statistical distributions of input parameters in geomechanics problems, three different concepts must be considered: uncertainties, variability and heterogeneities. These three concepts must be treated separately as they have various impacts on the rock mass behaviour and as different approaches must be used to tackle them. Uncertainties arise from the difficulty in measuring key geomechanical properties like rock stresses, rock modulus or rock strength. Any of these measurements is smudged with some error due to the sampling process, sample preparation or sensitivity and calibration of the measuring devices. This uncertainty is usually evaluated and reduced by acquiring repeated measurements during the development of a project (Figure 1). 


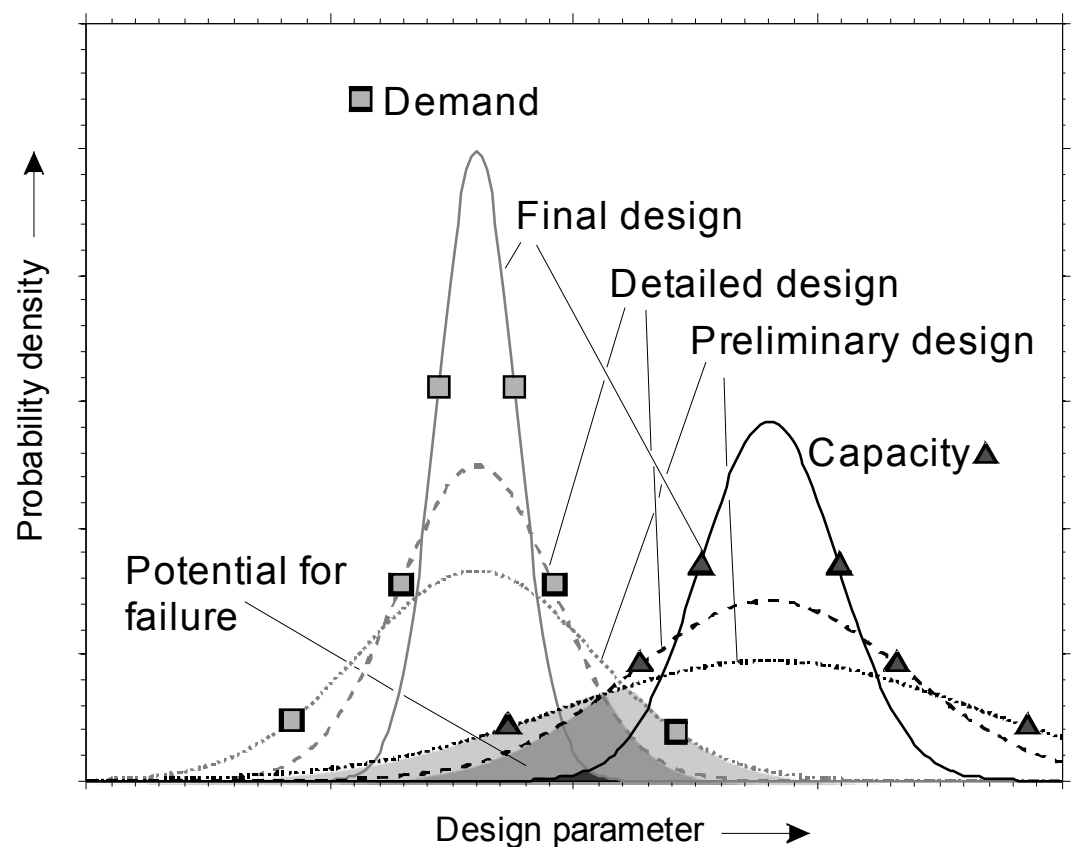

Figure 1 Illustration of the uncertainty reduction during the development of a project until the potential for failure is minimised to an acceptable level (after Hoek, 1992)

Considering a given design criteria, the probability of failure is given by the gray areas on Figure 1 combining it with the consequences of failure allows the computation of the risk of a given design (taking the standard definition of risk being probability of occurrence times consequence of occurrence) and to evaluate if this risk is acceptable. There is always some residual risk remaining even at the final design stage (darker grey on Figure 1). This residual risk needs to be handled by using mitigation measures, e.g. safety or worker exposure reduction procedures. The PEM/FEM method presented in this paper is particularly adapted to handle this kind of situation, i.e. it will allow one to track how uncertainties in the input parameters are propagated through the analyses and results in uncertainty in the design parameters. It allows the engineer to not limit the design to a single deterministic analysis with the most probable parameters (the mode of the distribution of Figure 1), but to evaluate the reliability of the design by considering the dispersion of the design parameters (e.g. Kaiser, 2010).

Variability is an inherent property of natural materials and rocks or rock masses are no exception to the rule. It arises from the various formation and transformation processes of rock and rock masses, including diagenesis, fractioned crystallisation, alteration through fluid circulation, metamorphism - all of which have local influence on the mechanical parameters of rock mass and rock masses. Due to this variability, rock mass properties will vary within a rock unit for example along the trace of a tunnel. Thus, a failure mechanism will affect more or less severely various locations along this tunnel.

Here again, the PEM/FEM approach presented in this paper is well-suited, and will for example allow the engineer to anticipate what percentage of a tunnel section will be affected by a failure mechanism for a given severity level. It will also allow for an evaluation of the range of severity of a given failure mechanism that should be anticipated and thereby permit the inclusion of flexibility in the design to handle the less probable but potentially more severe situation. Having an estimate of the distribution of the severity of a potential failure mechanism will also permit the optimisation of the support systems and to obtain a better estimation of the cost and thus the economical risk of a project. However, there are some limitations with this approach. Firstly, the severity of the failure mechanism evaluated must be continuous and monotonic: as will be illustrated later in this paper, if abrupt changes in behaviour occur the PEM method can be misleading. Thus, defining domains in which behaviour is similar and the severity of failure is continuous is critical. Finally, the wavelength of the variability must be relatively large compared to the engineering problem evaluated, so that homogeneous domains can be defined. In the case of short wavelength variations, the effect will be different and need to be treated, not as variability as defined here, but as heterogeneities as discussed in the following. 
Heterogeneities need to be treated separately as they will influence the severity of a failure mechanism and change the behaviour of the rocks or rock masses. For example, increasing modulus heterogeneities in a rock will promote the development of local tensile stress even in an overall compressive field which will affect the failure mode, e.g. change from shear mechanism to tensile dominated mechanism like spalling (e.g. Diederichs, 2007). The PEM/FEM approach proposed in this paper does not simulate the effect heterogeneities. Heterogeneities must be handled differently, either by the use of a classification system and equivalent homogeneous properties (Hoek and Brown, 1997) or by explicit modelling of the heterogeneities (e.g. Valley et al., 2010). The remainder of this paper will present an evaluation of the accuracy of the PEM/FEM approach by comparing its results with other probabilistic approaches. A detailed description of the method itself is presented next.

\section{PEM Method for numerical modelling}

In the simplest case, when closed-form analytical solutions are available for an analysis and when the input parameters are independent and uncorrelated, the propagation of errors can be approximated by using the following relation (first order Taylor series):

$$
\sigma_{y}=\left[\sum_{i=1}^{n}\left(\frac{\partial f}{\partial x_{i}}\right)^{2} \sigma_{i}^{2}\right]^{1 / 2}
$$

where $y=f\left(x_{1}, x_{2}, \ldots, x_{n}\right)$ and $\sigma_{n}$ the error on $x_{n}$. This relation requires that it is possible to extract a partial derivative for the function $f$. This is not always possible and obviously impossible when the solution to a problem is found by using a numerical method like FEM. The point estimate method proposed by Rosenblueth (1975) and modified later by others (e.g. He and Sällfors, 1994; Hong, 1995, 1998; Tsai and Franceschini, 2005), allows one to propagate error even if no closed-form analytical solution is available. PEM has been applied for geotechnical problems by others (e.g. Christian and Baecher, 1999; Joughin et al., 2000; Peschl and Schweiger, 2003; Schweiger and Peschl, 2005; Hammah et al., 2009). The principle of PEM is to compute solutions at various estimation points and to combine them with proper weighting in order to get an approximation of the distribution of the solution. The PEM used in this paper is the two-point estimate method for the first and second moment and uncorrelated variables. It needs $2^{n}$ evaluations of the solution, where $n$ is the number of random variables. The distribution of the solution for $y=f\left(x_{1}, x_{2}, \ldots, x_{n}\right)$ is given by:

$$
\begin{gathered}
\bar{y}=\sum_{i=1}^{2^{n}} w f_{i} \\
\sigma_{y}=\sqrt{\sum_{i}^{2^{n}} w f_{i}^{2}-\left[\sum_{i}^{2^{n}} w f_{i}\right]^{2}}
\end{gathered}
$$

where the weights $w$ are given by $1 / 2^{n}$. $f_{i}$ are successive evaluations of $f$ at the $2^{n}$ possible combinations of the random variables at the point estimate locations, i.e. at $\bar{x}_{n}-\sigma_{x_{n}}$ and $\bar{x}_{n}+\sigma_{x_{n}}$. In the solution presented here, all input variable and output variables are assumed to follow a normal distribution given by their mean $\bar{x}$ and standard deviation $\sigma_{x}$.

In order to illustrate how to use the PEM with FEM, the following example should be considered: the stress distribution around a circular opening has to be evaluated, but the estimation of the far field stresses $\left(S_{1}\right.$ and $S_{3}$ ) is uncertain. It can be assumed that this uncertainty can be captured by a normal distribution, i.e. a mean and a standard deviation (see modelling properties given in Table 1). 
Table 1 Input parameters for modelling

\begin{tabular}{lll}
\hline Input Parameter & Mean & $\begin{array}{l}\text { Standard } \\
\text { Deviation }\end{array}$ \\
\hline $\begin{array}{l}\text { Max. far field principal stress } \\
S_{1} \text { (horizontal) }\end{array}$ & $30 \mathrm{MPa}$ & $5 \mathrm{MPa}$ \\
$\begin{array}{l}\text { Min. far field principal stress } \\
S_{3} \text { (vertical) }\end{array}$ & $15 \mathrm{MPa}$ & $2 \mathrm{MPa}$ \\
Out of plane stress $S_{z}$ & $10 \mathrm{MPa}$ & $-^{-}$ \\
Young modulus $E$ & $20 \mathrm{GPa}$ & $-^{\text {a }}$ \\
Poisson ratio $v$ & 0.25 & $-^{\text {a }}$ \\
\hline
\end{tabular}

a - These variables are not considered as random variable and thus no standard deviation is defined for them.
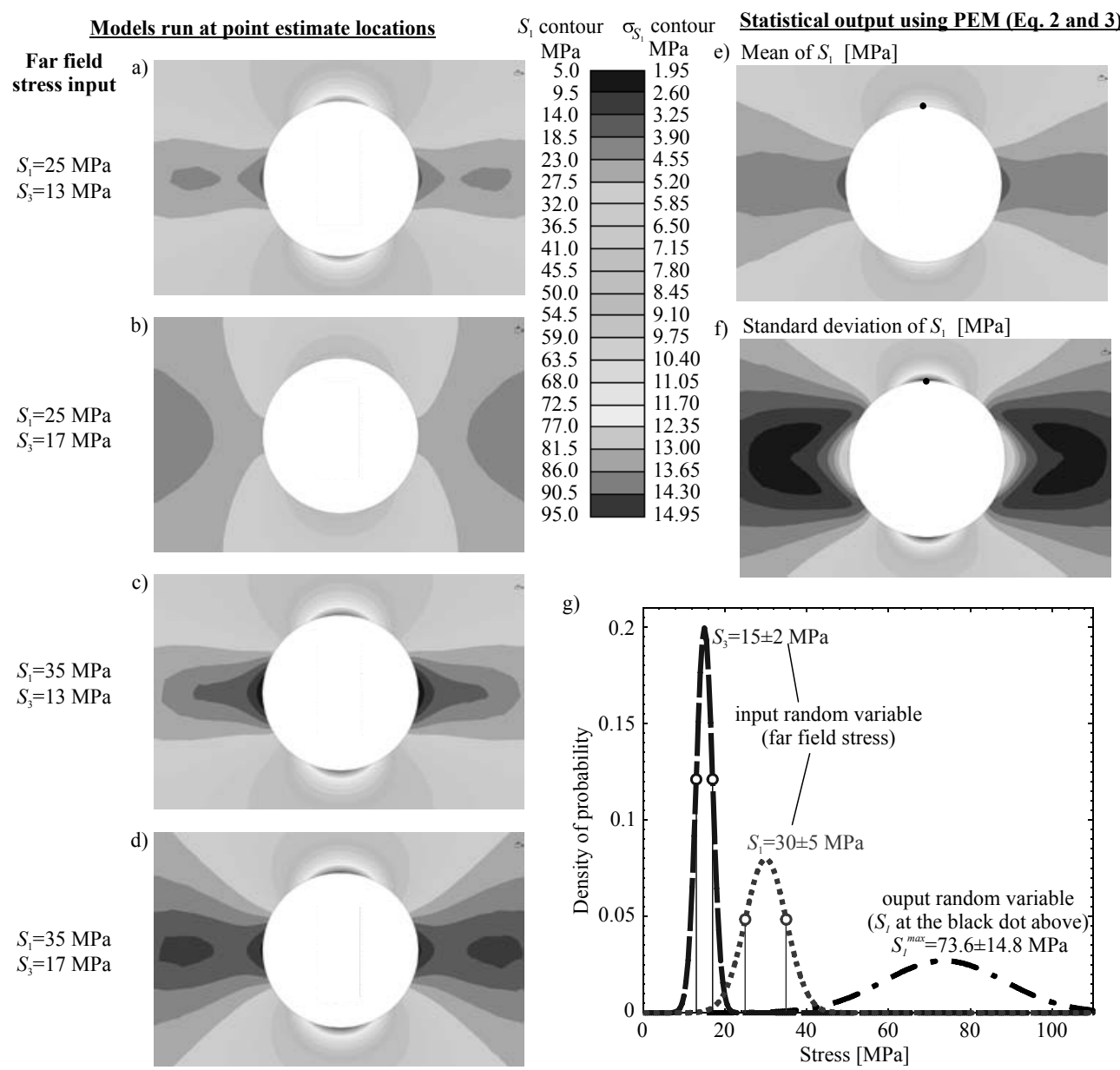

Figure 2 Example of PEM/FEM computation with parameters given in Table 1. a), b), c) and d) evaluation of the maximum principal stress $S_{1}$ (FEM elastic models) at the four combinations of the estimation points; e) and f) probabilistic output (mean and standard deviation) for $S_{1}$ obtained by combining the FEM results in the left with the PEM (Equations (2) and (3)); g) Probability density functions of the input ( $S_{1}$ and $S_{3}$, dashed line and dotted line) and the output of the analyses where principal stress is maximum $\left(S_{1}{ }^{\text {max }}\right)$ (see black dot for location on e and f)

In order to evaluate the uncertainty on some design parameter (assuming for example the maximum principal stress at the excavation boundary), four $\left(2^{2}\right.$, because there are two random variables, $S_{1}$ and $\left.S_{3}\right)$ models 
(Figure $2 \mathrm{a}$ to $2 \mathrm{~d}$ ) must be run assuming the following combinations of input for the far-field stresses: [ $S_{1}=25 \mathrm{MPa} ; S_{3}=13 \mathrm{MPa}$ ], [ $S_{1}=25 \mathrm{MPa} ; S_{3}=17 \mathrm{MPa}$ ], $\left[S_{1}=35 \mathrm{MPa} ; S_{3}=13 \mathrm{MPa}\right.$ ] and, $\left[S_{1}=35 \mathrm{MPa}\right.$; $S_{3}=17 \mathrm{MPa}$. These combinations are obtained by all possible combinations of mean \pm one standard deviation. The outputs of these models must then be combined using Equation (2) and Equation (3) in order to obtain the mean and standard deviation of the output design criteria (Figure 2e and 2f).

It is interesting to see that even in this simple case of elastic stresses around a circular opening, the pattern of uncertainty (see Figure 2f) is quite complex and not intuitive. The highest uncertainty is located where $S_{1}$ is the maximum while an area of low uncertainty arises in the $S_{1}$ far field direction at about one tunnel radius (darker area on Figure 2f). It is of interest to note that the deterministic solution for $S_{1}{ }^{\max }=75 \mathrm{MPa}$ and not 73.6 $\mathrm{MPa}$ as obtained from probabilistic approach.

In the following section, the robustness of the method will be evaluated by comparing results generated with the PEM/FEM approach with results obtained using Monte-Carlo simulations.

\section{Evaluation of PEM/FEM approach}

\subsection{Elastic stress distribution around a circular opening}

First we will further develop the simple elastic stress distribution around a circular opening presented in Section 2. The geometry and nomenclature associated with the problem is presented on Figure 3 . The tunnel radius $a$ will be $1 \mathrm{~m}$. In the following, we will particularly evaluate the stress conditions at a 'monitoring' point located at the coordinates $x=0.97 \mathrm{~m}$ and $y=0.91 \mathrm{~m}$, i.e. $r=1.33 \mathrm{~m}$ and $\theta=43^{\circ}$. The material properties considered are listed in Table 1.

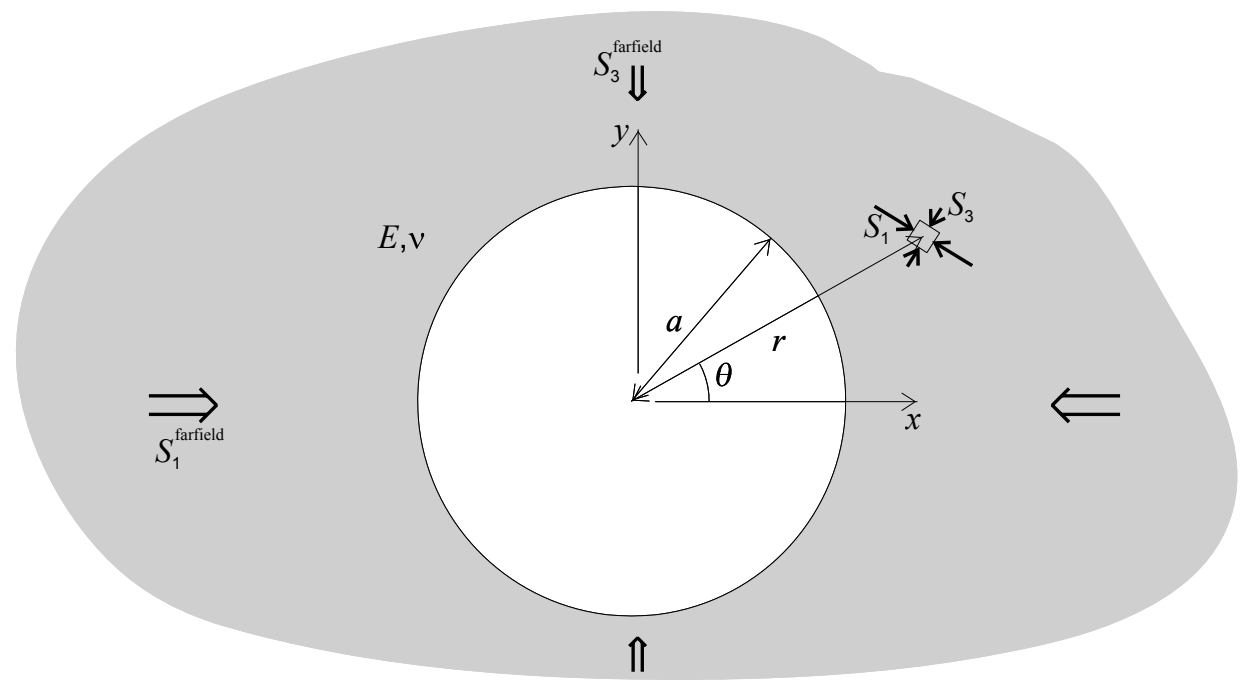

Figure 3 Geometry and nomenclature of the problem

In order to evaluate the robustness of the proposed PEM/FEM method, a comparison of the results obtained with the following alternative methods will be presented:

1. PEM/FEM method, as described previously.

2. A FEM (Phase2)/Monte-Carlo method: 1,000 FEM models were solved with random variable inputs. Two cases were considered: a) input random variables are uncorrelated and b) input random variables are directly correlated.

3. Solution using an analytical solution (Kirsch solution) and Monte-Carlo simulation: same approach as for 2. (1,000 models solved) with both correlated (a) and uncorrelated (b) case but an analytical solution for the stress redistribution around a circular opening is used.

4. Solution using an analytical (Kirsch solution) solution with the PEM (Rosenblueth). 
Comparisons of the results from these different methods, i.e. the distribution of the minimum and maximum principal stresses at the monitoring point, are presented in Table 2. A detailed example of the computation is illustrated in Figure 4, for the Monte-Carlo/FEM analyses assuming no correlation or direct correlation for the input random variables (far-field stresses), one thousand FEM solutions were computed in each case. The number of simulations $(1,000)$ was chosen for practicality (available computing time) and it was determined that with this number of simulations, the accuracy on the output stresses is better than $1 \mathrm{MPa}$.

Table 2 Outputs summary at the monitoring point for the various methods

\begin{tabular}{lllll}
\hline & \multicolumn{2}{c}{$\boldsymbol{S}_{\mathbf{1}}$} & \multicolumn{2}{c}{$\boldsymbol{S}_{3}$} \\
& Mean & $\boldsymbol{\sigma}$ & Mean & $\sigma$ \\
\hline Monte-Carlo analytical non-correlated & 37.50 & 5.20 & 6.71 & 1.34 \\
Monte-Carlo analytical correlated & 37.34 & 6.07 & 6.87 & 0.85 \\
Rosenblueth phase2 & 36.40 & 5.01 & 6.73 & 1.26 \\
Monte-Carlo phase2 correlated & 36.57 & 5.94 & 6.81 & 0.85 \\
Monte-Carlo phase2 non-correlated & 36.72 & 5.08 & 6.66 & 1.30 \\
Rosenblueth analytical & 37.21 & 5.13 & 6.74 & 1.30 \\
Deterministic solution & 37.06 & - & 6.89 & - \\
\hline
\end{tabular}

a)
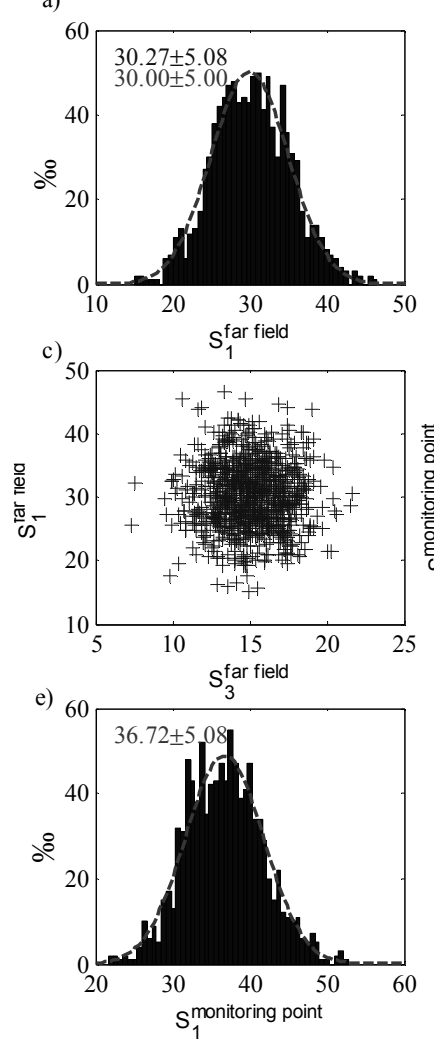

b)
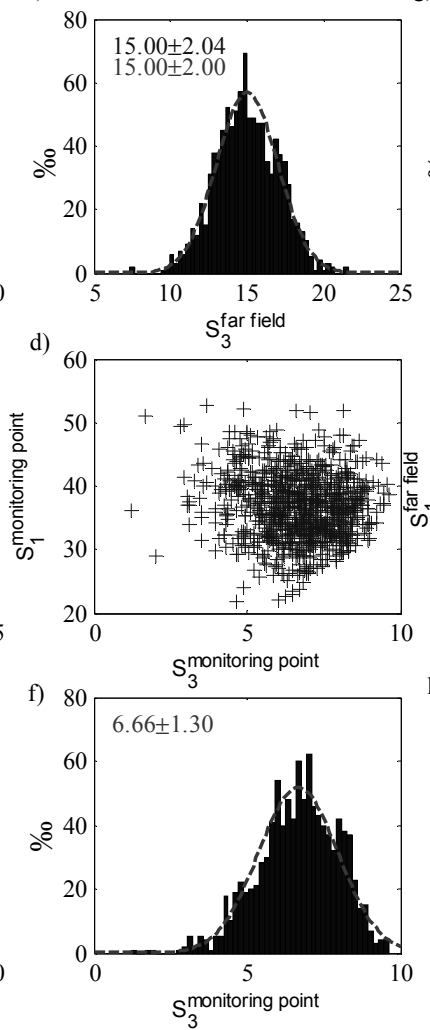

g)

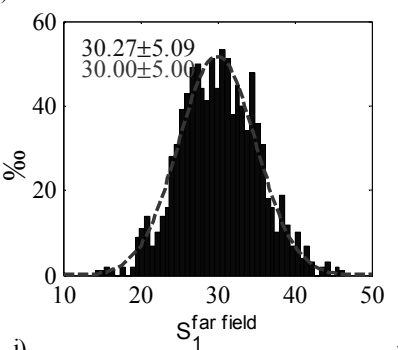

h)
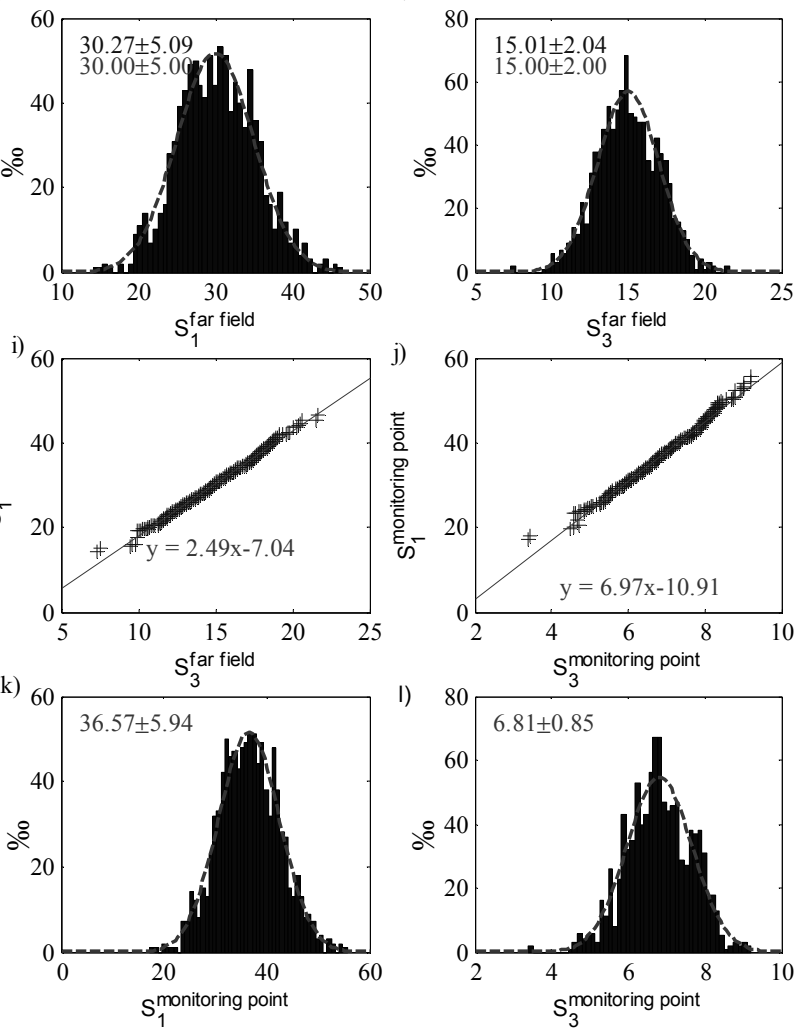

Figure 4 Inputs and outputs of Monte-Carlo analyses: a) to f) Far-field stresses are not correlated; g) to l) far-field stresses are correlated; a), b), g) and h) Distribution of the far-field stresses for the 1,000 FEM models (histogram) and actual distribution target (dashed curve); c) and i) scatter plot of the far-field stresses to show the correlation or non-correlation of the inputs; d) and j) scatter plot of the computed stresses show the correlation or noncorrelation of the outputs; e), f), $k$ ) and i) computed stress distribution at the monitoring point (histogram) and best normal distribution fit on the histograms (dashed curves) 
A comparison of the results from all the presented approaches is shown on Figure 5 and summarised in Table 2. All methods involving no correlation between the variables lead to very similar results (within $1 \mathrm{MPa}$ ) for both $S_{1}$ and $S_{3}$ at the monitoring point. However, the PEM/FEM approach is much more efficient in terms of computing resources as only 4 model runs are needed compared with 1,000 runs for the MonteCarlo approach.
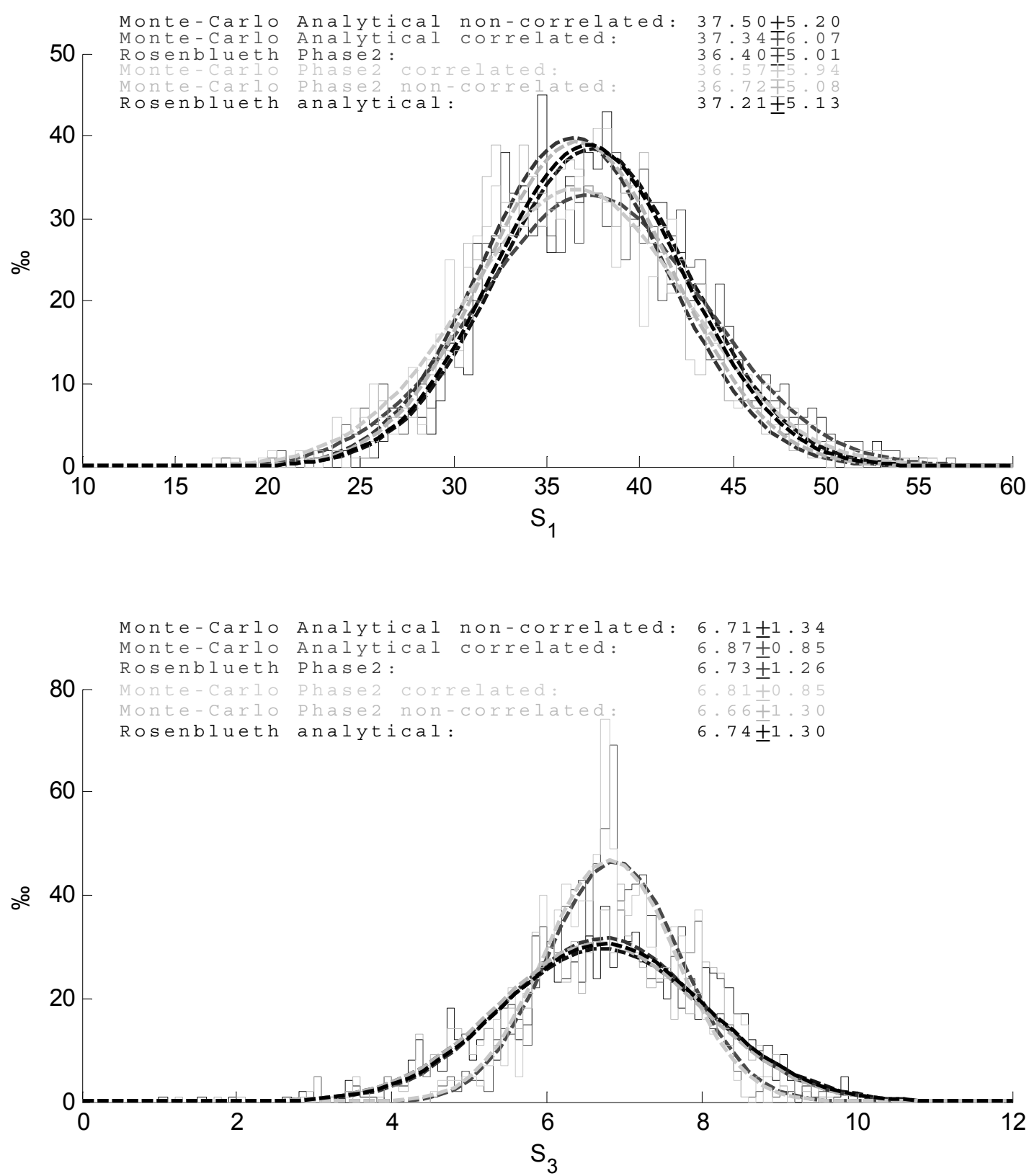

Figure 5 Graphical comparison of the output of the various analyses methods at the "monitoring" point. For Monte-Carlo simulations, the output histogram as well as the best fitted normal distribution is displayed

\subsection{Plasticity around a circular opening}

A non-elastic case, where failure related stress redistribution occurs, was also studied in a similar manner. The geometry of the case is identical to that for the elastic case (see Figure 3). However, this time the material model was non-linear (elasto-brittle-plastic). Three input parameters were varied: the maximum principal stress $\left(S_{1}=30 \pm 5 \mathrm{MPa}\right)$, the Young's Modulus $(E=20 \pm 2 \mathrm{GPa})$ and the cohesion $(c=10 \pm 3 \mathrm{MPa})$. 
Other inputs are kept identical to those of for the elastic case. In order to save computation time, the circular opening was entirely excavated within a single step. Phase 2 was used to solve this problem, using PEM and Monte-Carlo methods. In the Monte-Carlo method, two cases were investigated: without correlation between variables and with direct correlation between strength and modulus. The inputs for this modelling are presented in Figure 6.

Screen shots of few FEM models taken from the thousand models run are presented in Figure 7 and show the diversity of outputs (maximum principal stress is contoured and failure is indicated by crosses (x symbols and contoured by a black line) going from no yielding in some case to extensive yielding in others. It is important to note that the failure mode changes between each representation from elastic to yielding, depending on the selected parameters set. This leads to two families of results, some affected by yield and related stress redistribution, others purely elastic. The PEM is not designed for such situations and inaccuracies emerge as illustrated below.

A selection of the output from the probabilistic analyses is presented in Figure 8. Even if all the inputs were normally distributed, the distribution of the output from the Monte-Carlo analyses are sometimes complex, including skewness and kurtosis (e.g. Figure $8 \mathrm{~b}, 8 \mathrm{c}$ and $8 \mathrm{e}$ ). In these cases, the output differs significantly from a normal distribution as can be seen with the fitted normal distribution on Figure 8 (light gray dashed line).
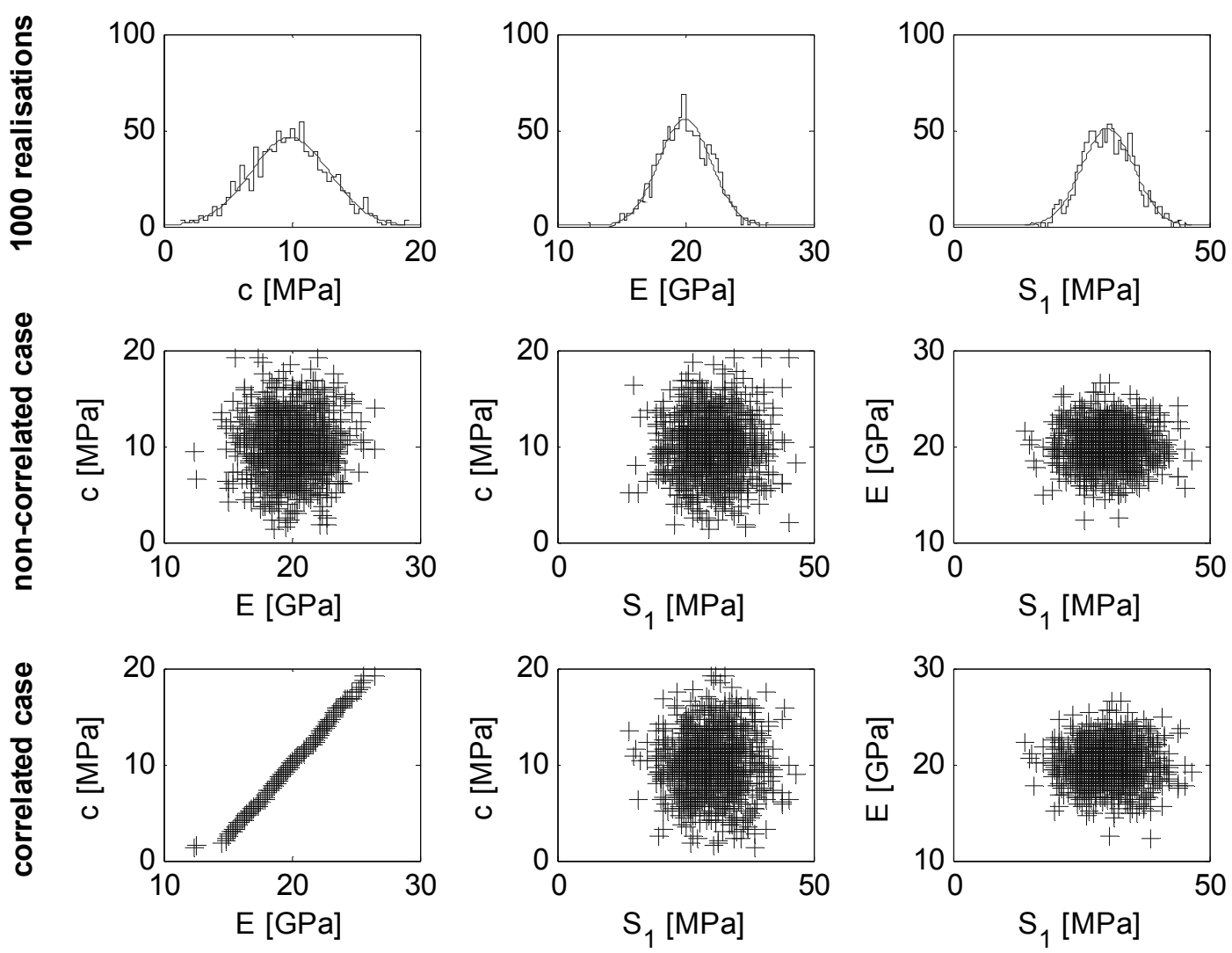

Figure 6 Input for the modelling of the plastic case: First row: distribution of cohesion, Young modulus and principal stress; Second row: scatter plot of variables for the modelling without correlation; Third row: scatter plot of variables for the models with correlation 





$S_{1}=29.305 \mathrm{MPa} E=22.195 \mathrm{GPa} \quad c=13.132 \mathrm{MPa}$

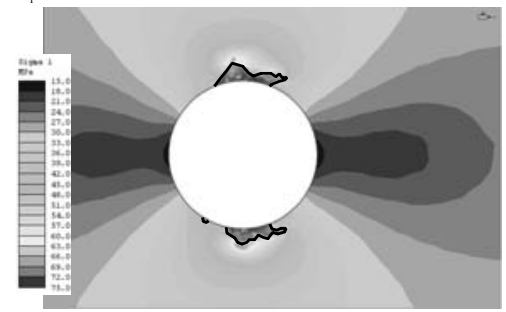

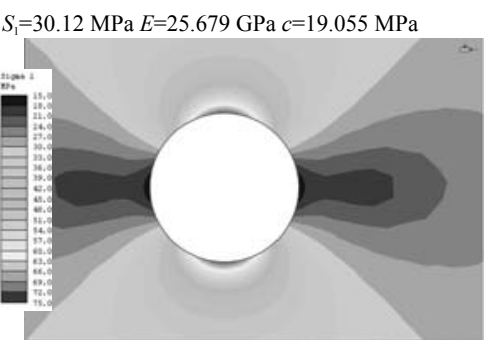

$S_{1}=37.422 \mathrm{MPa} E=16.73 \mathrm{GPa} c=5.0651 \mathrm{MPa}$



Figure 7 Some example of results drawn from the thousands of model runs for the Monte-Carlo analyses. $S_{1}$ contours are shown and yield is indicated by crosses. Specific value of the random variables for each runs are displayed above each models

a)

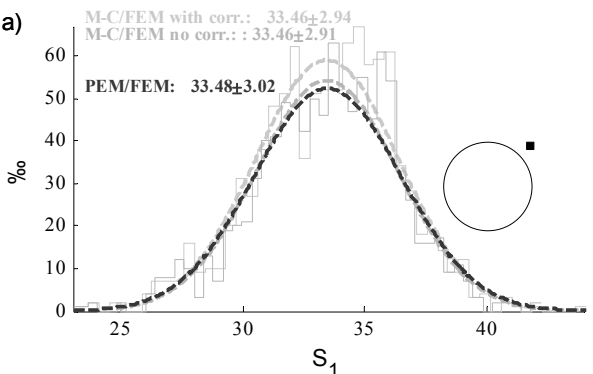

b)
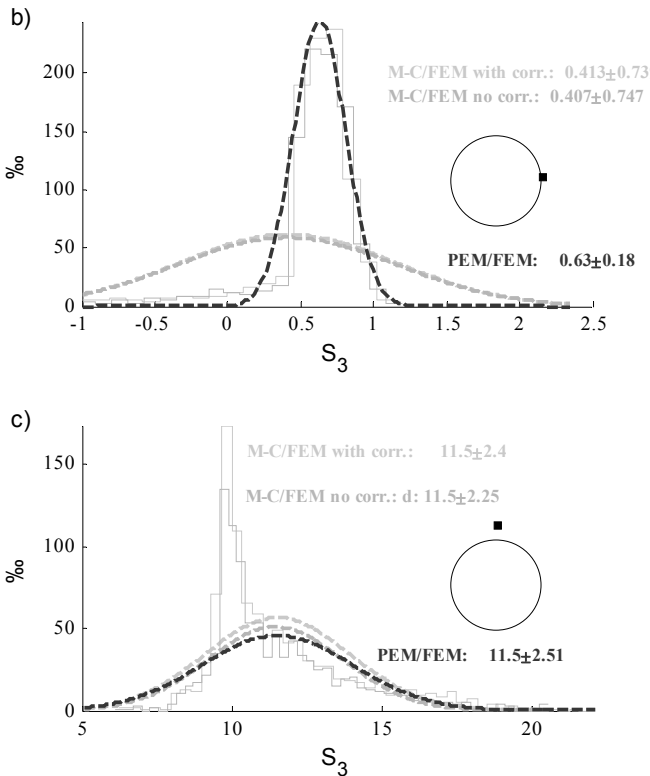

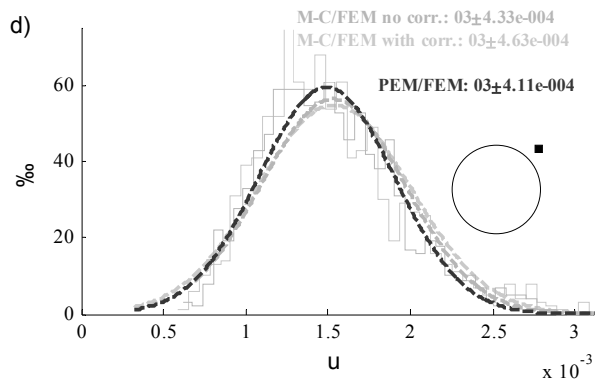

e)
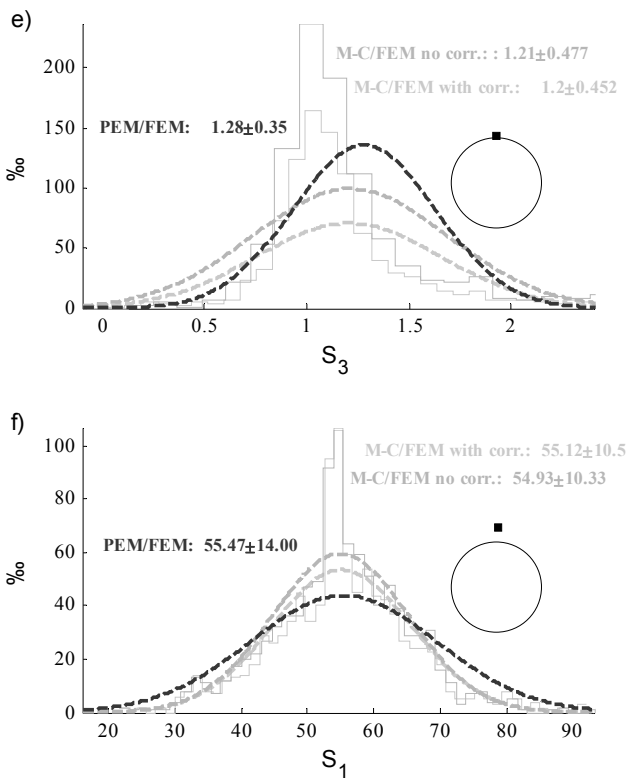

Figure 8 Comparison of probabilistic output for the plastic case using the various methods described in the text. Only a selection of outputs is presented. Inserted sketch show location each plot. $S_{1}$ : maximum principal stress [MPa], $S_{3}$ : minimum principal stress [MPa] and $u$ : displacement $[\mathrm{m}]$. The histograms are direct output of the Monte-Carlo simulations. The dashed light gray lines are normal best fit on the Monte-Carlo output. The darkest dashed line are the probability distribution obtain using the PEM/FEM approach 




Figure 9 Comparison of depth of yield obtained from the PEM approach with the ones obtained from Monte-Carlo (M-C) simulations

Comparison of the output from the Monte-Carlo simulations and the PEM approach (darkest lines on Figure 8) show that the PEM approach generally captures well the output distribution, particularly when the output distribution is close to a normal distribution. However, when the output distribution differs from a normal distribution, the PEM approach usually captures the mode properly but fails to capture the full range of the dispersion (e.g. Figure 8b). Specifically, it tends to underestimate the tails of the distribution. This occurs because the point estimate locations are relatively close to the mode $( \pm 1 \sigma)$ and thus do not contain information about the exceptional events (failure modes) of the tails of the distribution.

In some cases, the PEM approach fails to capture both the mode and the dispersion of the distribution obtained by Monte-Carlo simulation (Figure $8 \mathrm{c}$ and $8 \mathrm{e}$ ). This occurs when the output distribution reflects mixed behaviours mode, i.e. elastic or plastic behaviour in this case. If the monitoring points are located in the a zone where some of the stochastic realisations of the Monte-Carlo simulation generate plastic conditions while some others generate elastic conditions, the overall output will be a mixture of two failure behaviours that the PEM approach is not able to capture accurately.

In addition, the maximum depth of yield was recorded and a comparison of Monte-Carlo versus PEM is presented on Figure 9. While the PEM approach succeeds in getting the general trend, it fails in predicting the tail (probability $>10 \%$ ) of the distribution with high depth of failures. Here again the proximity of the estimation point from the mode in the chosen PEM scheme does not allow the proper capture of the tails.

\subsection{Application probabilistic estimation of the depth of yield along a tunnel}

Next, a case of a nominal $100 \mathrm{~m}$ long circular tunnel is investigated to account for the variability in rock mass quality and uncertainty in stresses. It is assumed that the variability of the geological strength index (GSI) is properly captured by a normal distribution with a mean of 65 and a standard deviation of five (refer to Cai and Kaiser (2006) to derive estimation of the variability of GSI). Also, uncertainty on the maximum principal stress of $30 \pm 5 \mathrm{MPa}$ is assumed. All other parameters are singled-value (deterministic). The depth of yield in shear is evaluated indirectly by considering the maximum shear strain. Figure 10a presents contours of maximum shear strain (mean case). It can be seen that the extent of yield is captured by the contour of $1 \mathrm{E}-003$ or $0.1 \%$ maximum shear strain for the selected stress and rock mass parameters.

The maximum shear strain radial profile in the back of the tunnel is presented on Figure 10b. If a deterministic approach was used with only the mean case being evaluated, the depth of yield is predicted to 
be as $0.32 \mathrm{~m}$ or $\mathrm{d}_{\mathrm{f}} / \mathrm{a}=1.32$. The error representing one standard deviation on the maximum shear strain obtained using the PEM/FEM approach is also shown. Combining the mean and the standard deviation allow the computation of a normal probability density function at each location (bell like surface on Figure 10c) from which the probability that the maximum shear strain exceeds 1E-003 (vertical plane on Figure 10c), i.e. that yield in shear occurs, can be evaluated (from integrating the violet hatched area). Thus a profile of the probability of yield can be computed (Figure 10d).

Considering that this probability of yield is controlled by variability along a $100 \mathrm{~m}$ tunnel section, one can predict that $50 \mathrm{~m}$ or $50 \%$ of this tunnel will have a depth of yield larger than $0.32 \mathrm{~m}$ or $\mathrm{d}_{\mathrm{f}} / \mathrm{a}>1.32$ (the result obtained by a deterministic analysis) and $50 \%$ of the tunnel will have a smaller depth of yield. The maximum depth of yield to be expected is $0.48 \mathrm{~m}$ and the minimum depth of yield will be about $0.05 \mathrm{~m}$. For about $10 \%$ of the tunnel the maximum depth of yield will exceed $0.40 \mathrm{~m}$ and $95 \%$ of the tunnel the depth of yield exceeding $0.10 \mathrm{~m}$. Only a few percent of the tunnel should be stable with no signs of failure. This example highlights the benefit of probabilistic analyses like the PEM/FEM approach, because it provides a deeper insight into the possible severity range of a given failure mechanism than a deterministic analyses. Furthermore, it is of practical value as the distribution of observed failures can be used to evaluate (or calibrate) the adopted failure model.

a)
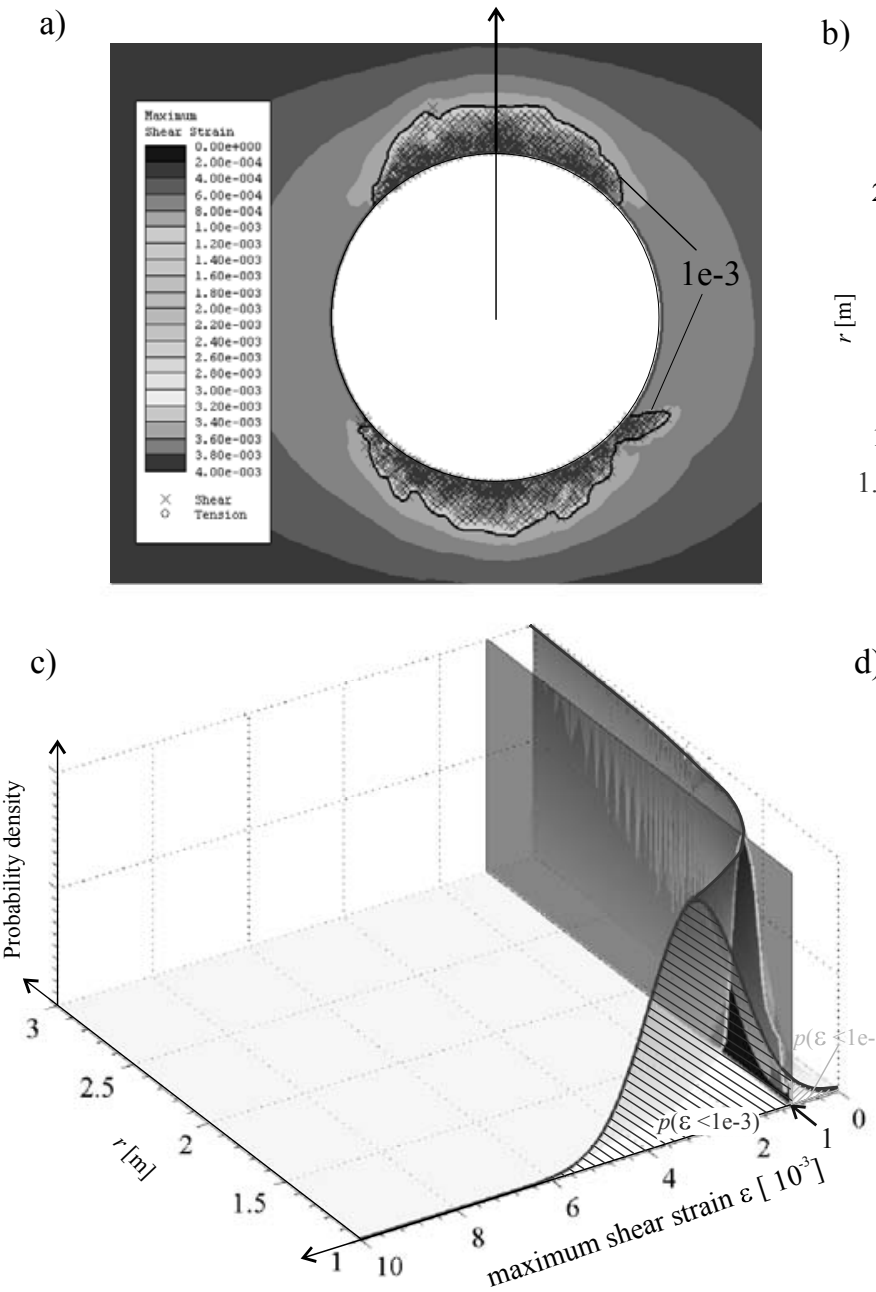

b)

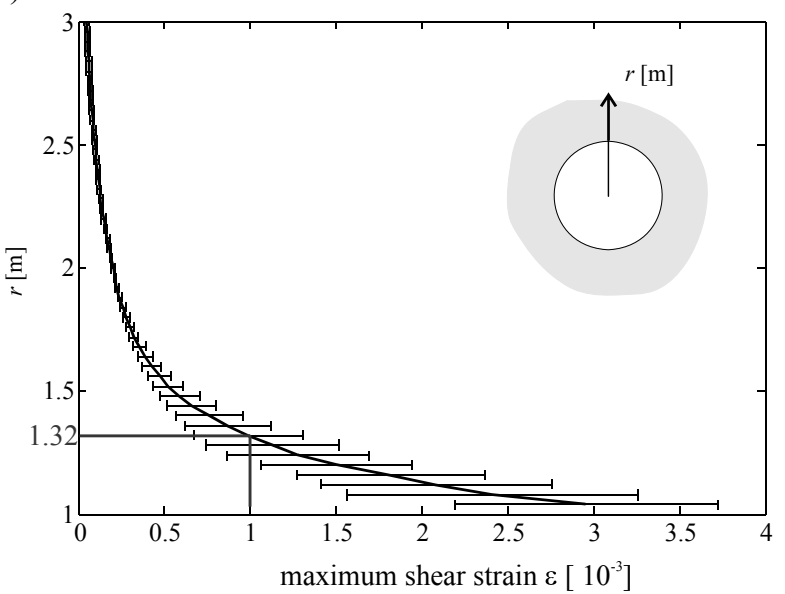

d)

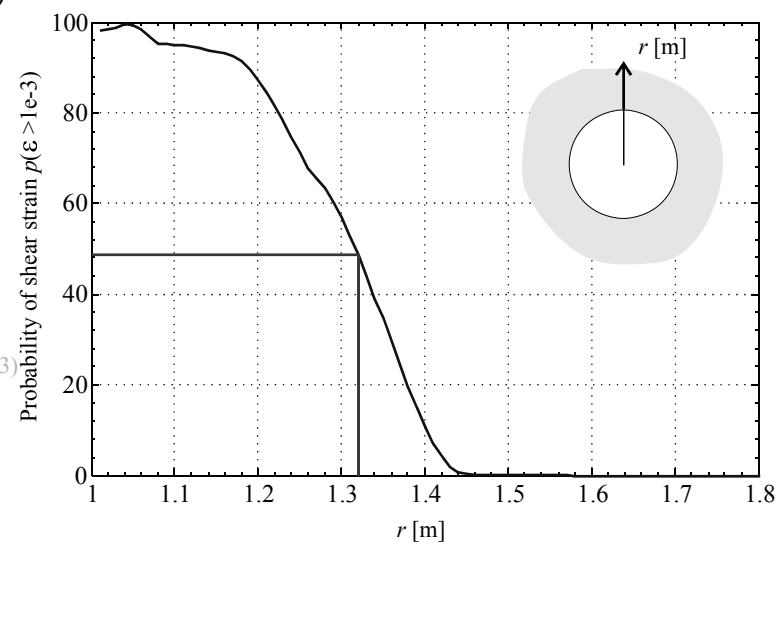

Figure 10 Application of probabilistic analyses for depth of yield using the PEM/FEM approach: a) maximum shear strain contour and element yielded in shear for the mean case; b) mean (black line) and standard deviations (error bar) on maximum shear strain in the back of the tunnel as a function of distance into the rock mass; c) representation of the density of probability as a function of the distance into the rock mass; d) probability of maximum shear strain limit of $0.1 \%$ 


\section{Conclusions}

The PEM/FEM approach evaluated in this paper presents an attractive opportunity to handle uncertainty and variability inherent in most geomechanical problems. The approximation by point estimates makes it computing power efficient and permits the performance of statistical analyses for problems for which other methods like Monte-Carlo simulation are not practicable. However, its simplicity brings some limitations. The PEM approach, as presented here without correlation, is based on normal and uncorrelated distribution. When a modelled case differs from these assumptions, and this is often the case in rock mechanics problems, the results can be inaccurate. Generally, the central tendency and some variability around it is well captured, but in many cases the tails may not be captured properly. Note that even when all the input variables follow the normal and uncorrelated assumptions, the output may not be normally distributed, and thus inaccuracy in the PEM/FEM output can be present. These limitations may be overcome by implementing more advanced versions of the point estimate method that handles second and third moments, as well as by using estimation points further away from the central tendency (e.g. Chang et al., 1995; Christian and Baecher, 2002).

When modelling involves behaviour discontinuities, as for example when transiting from elastic to plastic domains, the point estimate method shows further limitations and does not accurately capture the distribution of the design criteria. For this reason, it is recommended to test the effect of a limited number of random variables at a time. This will not only save computation time and allow deeper exploration of the possible outcomes but will also permit a better understanding and control over the potential bias introduced by the PEM/FEM approach. In addition to the outputs obtained using the proposed PEM/FEM approach, it is recommended to manually run some extreme cases of the targeted distributions in order to evaluate if it captures the tails of the output distribution properly. Another aspect which need to be tackled in order to make probabilistic analyses practicable in the field of rock mechanics is to develop the techniques and knowledge to properly characterise probabilistic inputs for the analyses (similar to what is done in the field of soil mechanics, e.g. Phoon and Kulhawy (1999)), including estimation of non-obvious parameters like variables correlations.

In summary, when used with awareness of the assumptions and potential limitations, the PEM/FEM approach offers an attractive and very efficient way of considering uncertainty in FEM analyses. It should lead to a broader use of the probabilistic approach in the mining industry and a better assessment of the reliability level of the design of underground openings.

\section{Acknowledgements}

This research is supported by CEMI's industrial sponsors, the Government of Ontario through its Ministry of Research and Innovation as well as by NSERC (Natural Sciences and Engineering Research Council of Canada).

\section{References}

Cai, M. and Kaiser, P.K. (2006) Rock mass characterization and rock mass property variability considerations for tunnel and cavern design, in Proceedings 4th Asian Rock Mechanics Symposium (ARMS 4), C.F. Leung, and Y.X. Zhou (eds), Paper 144, Singapore.

Chang, C-H., Tung, Y-K. and Yang, J-C. (1995) Evaluation of probability point estimate methods, Applied Mathematical Modelling, doi:10.1016/0307-904X(94)00018-2, Vol. 19 (2), pp. 95-105.

Christian, J.T. and Baecher, G.B. (1999) Point-estimate method as numerical quadrature, Journal of Geotechnical and Geoenvironmental Engineering, Vol. 125 (9), 779 p.

Christian, J.T. and Baecher, G.B. (2002) The point-estimate method with large numbers of variables, International Journal for Numerical and Analytical Methods in Geomechanics, doi:10.1002/nag.256, Vol. 26, No. 15, pp. 1515-1529.

Diederichs, M.S. (2007) The 2003 Canadian geotechnical colloquium: Mechanistic interpretation and practical application of damage and spalling prediction criteria for deep tunnelling, Canadian Geotechnical Journal, Vol. 44 (9), pp. 1082-1116.

Hammah, R.E., Yacoub, T.E. and Curran, J.H. (2009) Numerical modeling of slope uncertainty due to rock mass jointing, in International Conference on Rock Joints and Jointed Rock Masses '09.

Harr, M.E. (1987) Reliability-Based Design in Civil Engineering, McGraw-Hill, New York. 
Harr, M.E. (1989) Probabilistic estimates for multivariate analyses, Applied Mathematical Modelling, doi:10.1016/ 0307-904X(89)90075-9, Vol. 13(5), pp. 313-318.

He, J. and Sällfors, G. (1994) An optimal point estimate method for uncertainty studies, Applied Mathematical Modelling, doi:10.1016/0307-904X(94)90327-1, Vol. 18 (9), pp. 494-499.

Hoek, E. (1992) When is a design in rock engineering acceptable? - 1991 Müller Lecture, in Proceedings 7th Congress Int. Soc. Rock Mech., Aachen: A.A. Balkema, pp. 3-25.

Hoek, E. and Brown, E.T. (1997) Practical estimates of rock mass strength, International Journal of Rock Mechanics and Mining Sciences, doi:10.1016/S1365-1609(97)80069-X, Vol. 34 (8), pp. 1165-1186.

Hong, H.P. (1995) An optimal point estimate method for uncertainty studies, Applied Mathematical Modelling, Vol. 19, No. 8, pp. 508-509.

Hong, H.P. (1998) An efficient point estimate method for probabilistic analysis, Reliability Engineering and System Safety, Vol. 59 (3), pp. 261-267.

Joughin, W.C., Swart, A.H. and Wesseloo, J. (2000) Risk based chromitite pillar design - part 2: Non-linear modelling, in Proceedings South African National Institute of Rock Engineers Symposium: Keeping it in the Bushveld and Advances in Support Technology, Rustenburg, South Africa.

Kaiser, P.K. (2010) How highly stressed brittle rock failure impacts tunnel design, In European Rock Mechanics Symposium (EUROCK) Lausanne, Balkema.

Peschl, G.M. and Schweiger, H.F. (2003) Reliability analysis in geotechnics with finite elements, comparison of probabilistic, stochastic and fuzzy set methods, in Proceedings of ISITPA'03, Lugano, Switzerland.

Phoon, K-K. and Kulhawy, F.H. (1999) Characterization of geotechnical variability, Canadian Geotechnical Journal, doi:10.1139/cgj-36-4-612, Vol. 36 (4), pp. 612-624.

Rosenblueth, E. (1975) Point estimates for probability moments, in Proceedings of the National Academy of Sciences, Vol. 72 (10), pp. 3812-3814.

Schweiger, H. and Peschl, G. (2005) Reliability analysis in geotechnics with the random set finite element method, Computers and Geotechnics, doi: 10.1016/j.compgeo.2005.07.002, Vol. 32 (6), pp. 422-435.

Tsai, C.W. and Franceschini, S. (2005) Evaluation of probabilistic point estimate methods in uncertainty analysis for environmental engineering applications, Journal of Environmental Engineering, Vol. 131, pp. 387-395.

Valley, B., Suorineni, F.T. and Kaiser, P.K. (2010) Numerical analyses of the effect of heterogeneities on rock failure process, in ARMA conference, paper no. 10-648, Salt Lake City. 
